Original

\title{
Expression of Wilms' tumor 1 (WT1) in ameloblastomas
}

\author{
Ronell Bologna-Molina1), Yasunori Takeda2), Takahisa Kuga ${ }^{3)}$, Naoyuki Chosa4), \\ Masae Kitagawa ${ }^{5}$, Takashi Takata6), Akira Ishisaki4), and Toshinari Mikami2) \\ 1)Molecular Pathology Area, School of Dentistry, University of the Republic, Montevideo, Uruguay \\ 2)Division of Anatomical and Cellular Pathology, Department of Pathology, Iwate Medical University, \\ Iwate, Japan \\ 3)Department of Biochemistry and Molecular Biology, Kyoto Pharmaceutical University, Kyoto, Japan \\ 4)Division of Cellular Biosignal Sciences, Department of Biochemistry, Iwate Medical University, Iwate, Japan \\ ${ }^{5)}$ Center of Oral Clinical Examination, Hiroshima University Hospital, Hiroshima University, Hiroshima, Japan \\ 6)Department of Oral and Maxillofacial Pathobiology, Institute of Biomedical and Health Sciences, \\ Hiroshima University, Hiroshima, Japan
}

(Received October 14, 2015; Accepted March 23, 2016)

\begin{abstract}
The Wilms' tumor 1 gene (WT1) was originally isolated and described as the gene responsible for Wilms' tumor. Although there is growing evidence linking the overexpression of WT1 to tumorigenesis, no reports on ameloblastoma are available at present. The aim of this study was to examine the expression of WT1 in various histological subtypes of ameloblastoma tissue specimens and in human ameloblastoma cell lines. Immunohistochemical analyses were performed on a total of 168 cases of ameloblastoma, one case of ameloblastic carcinoma, and five cases of tooth germs (control). Three immortalized human dental epithelial cell lines (HAM1, HAM2, and HAM3) derived from the same ameloblastoma patient were used for reverse transcription-polymerase chain reaction (RT-PCR) and western blot assays. The tooth germs did not express WT1 (0\%), and more than half of the ameloblastoma cases showed

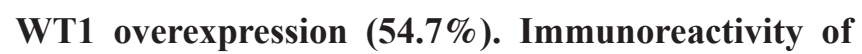
solid-type ameloblastoma (76.1\%) was more evident than that of unicystic-type ameloblastoma (40.9\%). The expression level of WT1 mRNA in HAM2 was
\end{abstract}

Correspondence to Dr. Ronell Bologna-Molina, Department of Molecular Pathology, Facultad de Odontología, Universidad de la República (UDELAR), Las Heras 1925, Montevideo, Uruguay Fax:+598-24873048 E-mail: ronellbologna@hotmail.com

doi.org/10.2334/josnusd.15-0546

DN/JST.JSTAGE/josnusd/15-0546 higher than that in HAM1 (moderate) and HAM3 (weak), showing the heterogeneity of tumor cells. The WT1 protein was strongly detected in HAM2 and minimally detected in HAM1 and HAM3. Our results suggest that WT1 expression influences the pathogenesis of ameloblastoma by varying its expression level in different histological types.

(J Oral Sci 58, 407-413, 2016)

Keywords: Wilms' tumor 1; WT1; ameloblastoma; heterogeneity.

\section{Introduction}

Odontogenic tumors are heterogeneous lesions derived from the epithelial, ectomesenchymal, and/or mesenchymal elements of tooth-forming tissues and can pose a significant diagnostic and therapeutic challenge (1). They are one of the main causes of tissue destruction in the oral and maxillofacial regions.

Ameloblastoma is a benign lesion, and is the most prevalent amongst epithelial odontogenic tumors. However, these tumors exhibit various biological behaviors ranging from simple cystic expansion to aggressive solid masses characterized by local invasiveness, a high risk of recurrence, and even malignant transformation. In 2005, the World Health Organization classified ameloblastomas as solid/multicystic (SA), unicystic (UA), extraosseous/peripheral (PA), and desmoplastic (DA) 
(1).

The Wilms' tumor 1 gene (WT1), located at chromosome $11 \mathrm{p} 13$, was originally isolated and characterized as being responsible for Wilms' tumor or nephroblastoma, which typically occurs in pediatric patients and rarely occurs in adults (2). Although WT1 was originally described as a tumor suppressor gene, it has now been suggested that it performs an oncogenic function instead (3-5).

The WT1 protein is detected in various types of malignancies including oral squamous cell carcinoma (OSCC), gastrointestinal and pancreatico-biliary tumors, urinary and genital tumors, breast tumors, brain tumors, malignant melanoma, soft tissue sarcoma, and osteosarcoma $(3,6-8)$, and its expression level serves as a significant prognostic factor.

Clinical trials examining WT1 peptide-based cancer immunotherapy by focusing on various types of tumors (9-15) have proven its clinical efficacy and safety. Thus, this procedure is considered to be a potential new treatment for various tumors with WT1 over-expression (5). However, the expression of WT1 in ameloblastoma, the treatment strategy for which is limited to surgical resection, remains undetermined.

Therefore, the aim of this study was to investigate the expression of WT1 in various histological types of ameloblastoma to allow examination of the feasibility of using WT1 peptide-based immunotherapy in such patients. The study was completed using a large series of ameloblastoma tissue specimens and three immortalized dental epithelial cell lines derived from human ameloblastomas.

\section{Materials and Methods}

This study used three immortalized human dental epithelial cell lines (HAM1, HAM2, and HAM3) derived from the same ameloblastoma patient and established in Iwate Medical University School of Dentistry, Morioka, Japan (16). K562 cells (human leukemia cell line) obtained from the Riken Bio Resource Center (Ibaraki, Japan) were used as a positive control.

This study also included a total of 168 cases of ameloblastoma, one ameloblastic carcinoma, and five tooth germs (one in the cap stage and four in the bell stage, which served as a control) retrieved from three facilities of the Department of Oral Pathology in Mexico and the Department of Histology and Molecular Pathology at the University of the Republic in Uruguay. The specimens were harvested from non-decalcified portions of the tumors, fixed in $10 \%$ buffered formalin, and embedded in paraffin. All cases were evaluated and classified according to the most recent World Health Organization classification of tumors (1).

The study was approved by the Ethics Committee of the School of Dentistry, University of the Republic (UDELAR) Montevideo Uruguay, number 0091900000113-14.

\section{Immunohistochemistry}

This study used the immunochemistry techniques reported by us in a previous study (17). In brief, $2-\mu \mathrm{m}$-thick tissue sections were incubated with a primary antibody [monoclonal antibody was used against WT1 (M3561 Clone 6F-H2; 1:100 dilution, Dako, Carpinteria, CA, USA)] for $45 \mathrm{~min}$. For the negative controls, the primary antibody was replaced with PBS.

Evaluation of WT1 positivity was performed by a single examiner who was blinded to the patient's clinical data. Quantification was performed visually using an optical microscope (Eclipse Ci-L, Nikon, City, Japan) within 10 high-power fields/slide and at $40 \times$ magnification, and scored as follows: 0 ("essentially no staining") for negative or positive immunohistochemical staining of $<5 \%$ of the cells; + ("weak-moderate") for staining of 5 to $50 \%$ of cells, and ++ ("strong positive") for $>50 \%$ positive staining. The mean staining intensity was then calculated.

\section{Western blotting}

Western blotting was performed using the standard procedure described in our previous study (7). The membrane was incubated with a primary anti-WT1 (1:2000, M3561; Dako) antibody and an anti-GAPDH (1:2000, IMG5143A; Imgenex, San Diego, CA, USA) antibody, which was used as a loading control for normalization.

\section{RNA preparation and reverse transcription-} polymerase chain reaction analysis (RT-PCR)

This procedure was performed as previously described (7). Total RNA was collected from confluent cultured cells using an RNeasy Mini Kit (Qiagen Inc., Valencia, CA, USA) according to the manufacturer's instructions, and complementary DNA (cDNA) was synthesized. The sequences of GAPDH and WT1 primers used were as follows: GAPDH, forward primer 5'-CAATGACCCCTTCATTGACC-3' (exon 2); reverse primer 5'-GACAAGCTTCCCGTTCTCAG-3' (exon 3); WT1, forward primer 5'-AGGGTACGAGAGCGATAACCACAC-3' (exon 6); and reverse primer 5'-TCAG ATGCCGACCGTACAAGA-3' (exon 7). K562 (human leukemia cell line) total RNA (AM7832; Applied Biosystems) was used to synthesize cDNA, which was 


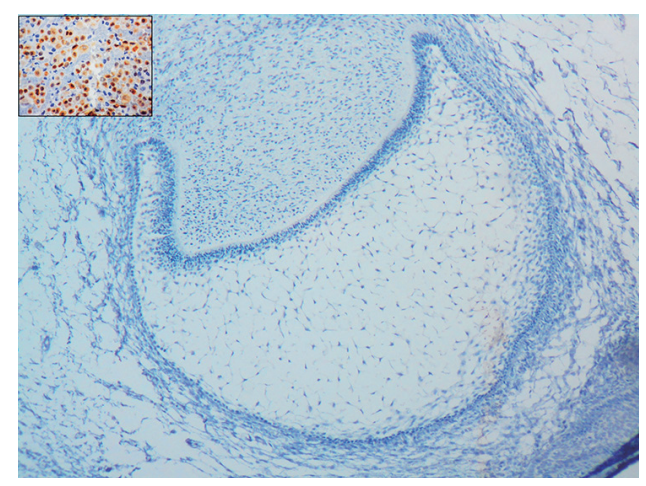

Fig. 1 Tooth germ demonstrating no WT1 protein expression (magnification 100×). Mesothelioma was used as the positive control (upper left corner).

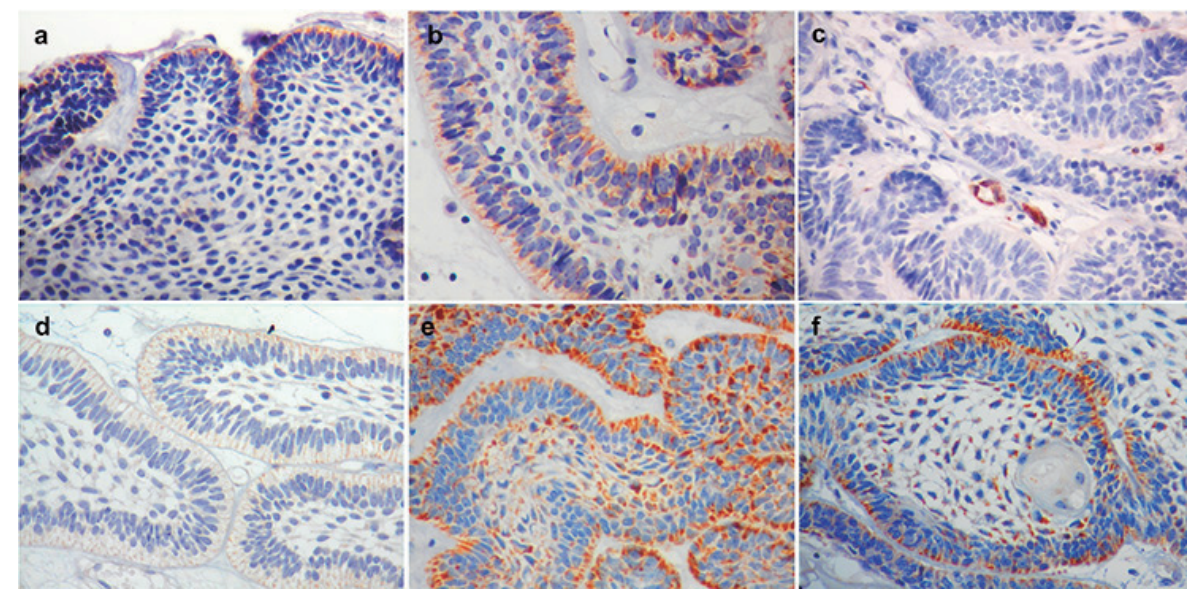

Fig. 2 Differential WT1 expression in solid ameloblastomas, magnification 200×. a and b) The ameloblastomas exhibit increased WT1 protein expression in ameloblast-like basal cells, whereas the stellate reticulum-like cells primarily exhibit medium or low WT1 expression. c) Some cases were negative (-) for WT1 protein, whereas endothelial cells of blood capillaries were positive (internal control). d) Weak/moderate (+) WT1 expression in follicular ameloblastoma. e) Strongly positive (++) WT1 protein expression in plexiform ameloblastoma. f) Strongly positive $(++)$ WT1 protein expression in acanthomatous ameloblastoma.

used as the positive control (10), while that synthesized from total RNA extracted from normal human epidermal keratinocytes (NHEK, KK-4001; Kurabo, Tokyo, Japan) was used as the negative control.

\section{Statistical analyses}

The data was analyzed using the Pearson's $\chi^{2}$ test and Kruskal-Wallis test. The Mann-Whitney test was utilized to detect differences between the different types of ameloblastomas and WT1 expression. The results were considered significant if $P<0.05$, and all statistical analyses were performed on SPSS 12.0 (SPSS Professional Statistics; SPSS Inc., Chicago, IL, USA).

\section{Clinicopathological data}

\section{Results}

The predominant type of tumor was UA $(n=93 / 169 ; 55 \%$ of all cases), 58 of which were the intraluminal subtype,
27 were the mural subtype, and eight were the luminal subtype. The SA type accounted for $42 \%$ of all cases (71/169), 48 of which were plexiform, 16 were follicular, and seven were acanthomatous. Only $2.4 \%$ of cases were DA (4/169), and $0.6 \%$ of cases were ameloblastic carcinoma (1/169).

The male:female (M:F) ratio was 1:1, with 84 males and 85 females, and the age range was 2-79 years (mean: 39.2 years). A clear predominance of mandibular localization was observed in this study, with $92 \%$ of the cases exhibiting lower jaw involvement. In addition, most of these tumors involved the posterior area and measured between 3-6 cm (mean size of mandibular tumors: 5.1 $\mathrm{cm})$.

\section{Immunohistochemistry}

All five tooth germs were negative for WT1 (Fig. 1). The ameloblastomas exhibited higher WT1 expres- 


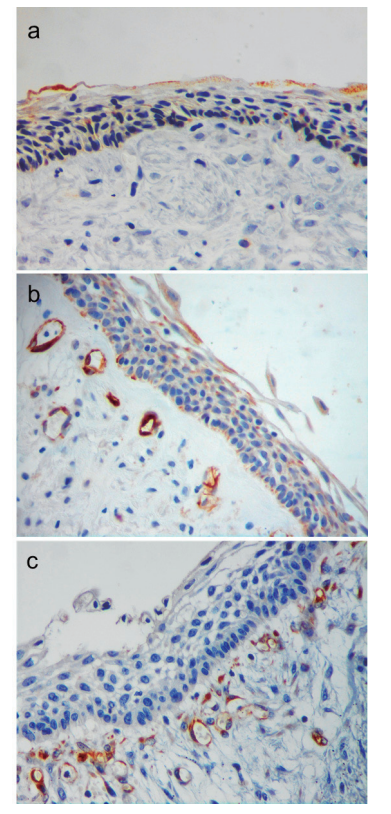

Fig. 3 Differential WT1 in unicystic ameloblastomas, magnification 200×. a and b) Strongly positive $(++)$ WT1 protein expression in unicystic ameloblastoma. c) Negative WT1 protein expression in the cystic ameloblastic epithelium and endothelial cells of blood capillaries as internal controls.
Table 1 WT1 expression in the different ameloblastoma subtypes

\begin{tabular}{lrcccc}
\hline $\begin{array}{l}\text { Ameloblastoma } \\
\text { histological type }\end{array}$ & $n$ & $\begin{array}{c}\text { Negative } \\
n(\%)\end{array}$ & $\begin{array}{c}+ \\
n(\%)\end{array}$ & $\begin{array}{c}++ \\
n(\%)\end{array}$ & $P$ values \\
\hline Solid/Multicystic & 71 & $17(23.9)$ & $36(50.7)$ & $18(25.4)$ & $0.01^{*}$ \\
PSA & 48 & $13(27.1)$ & $25(52.1)$ & $10(20.8)$ & \\
FSA & 16 & $4(25)$ & $9(56.2)$ & $3(18.7)$ & \\
ASA & 7 & $0(0)$ & $2(28.5)$ & $5(71.4)$ & \\
Unicystic & 93 & $55(59.1)$ & $34(36.6)$ & $4(4.3)$ & $0.03^{*}$ \\
LUA & 8 & $2(25)$ & $5(62.5)$ & $1(12.5)$ & \\
IUA & 58 & $40(69)$ & $16(26)$ & $2(5)$ & \\
MUA & 27 & $13(48.1)$ & $14(48.1)$ & $1(3.7)$ & \\
Desmoplastic & 4 & $4(100)$ & $0(0)$ & $0(0)$ & \\
Ameloblastic carcinoma & 1 & $0(0)$ & $1(100)$ & $0(0)$ & \\
Tooth germs & 5 & $5(100)$ & $0(0)$ & $0(0)$ & \\
\hline
\end{tabular}

Differences in the degree of WT1 expression in different ameloblastoma histological types and subtypes. Solid/Multicystic vs Unicystic $P=0.000 * *$ * Kruskal-Wallis test, **Mann-Whitney test. IUA: intraluminal unicystic ameloblastoma; LUA: luminal unicystic ameloblastoma; MUA: mural unicystic ameloblastoma; FSA: follicular solid ameloblastoma; ASA: acanthomatous solid ameloblastoma; PSA: plexiform solid ameloblastoma. sion in ameloblast-like basal cells, whereas the stellate reticulum-like cells primarily exhibited medium or low expression. WT1 protein expression was found exclusively in the cytoplasm of tumor cells. The endothelial cells of the blood capillaries were positive (internal control for immunohistochemistry) in all cases (Figs. 2, 3).

More than half of the ameloblastomas showed WT1 overexpression (54.7\%), and the SA type exhibited more evident immunoreactivity $(76.1 \%)$ than the UA type $(40.9 \%, P<0.05$; Table 1 and Figs. 2, 3). Some minor differences were detected between the SA and UA subtypes (Table 1).

All DA samples were negative for WT1. Because of the rarity of this tumor, only one ameloblastic carcinoma was included in this study, and this specimen was positive for WT1.

The categorization of positive immunostained samples into two groups, namely, weakly/moderately positive $(+)$ or strongly positive $(++)$, showed that all types and subtypes predominantly exhibited weak/moderate positivity, with the exception of the solid acanthomatous subtype (Fig. 2), which exhibited $71.4 \%$ strong positivity (Table 1).

No statistically significant differences in WT1 immunohistochemical staining were observed by clinical variables such as age, sex, location, size, and recurrence (Table 2).

\section{WT1 expression in cell lines}

The expression of WT1 protein was examined using western blotting. It was strongly detected in HAM2, and slightly detected in HAM1 and HAM3 (Fig. 4). The expression level of WT1 mRNA in HAM2, examined by RT-PCR, was higher than that in HAM1 (moderate) and HAM3 (weak; Fig. 5).

\section{Discussion}

The present study examined the expression of WT1 in various histological subtypes of ameloblastoma tissue specimens and in human ameloblastoma cell lines established from the same patient. Although all tooth germs were negative for WT1, more than half of the ameloblastoma tissue samples showed WT1 overexpression. Moreover, although tumor cells of the tissue samples may be influenced by stroma expressing WT1, the cell lines expressed WT1 without being influenced by any other factors. These findings suggest a relationship between WT1 and the pathogenesis of ameloblastoma. Based on the results that ameloblast-like basal cells exhibited strong positivity, stellate reticulum-like cells exhibited medium or low positivity in the tumor nests, and the 
Table 2 Clinicopathological features and WT1 expression

\begin{tabular}{|c|c|c|c|c|c|}
\hline Factor & Negative & + & ++ & Total & $P$ value \\
\hline \multicolumn{6}{|l|}{ Age (years) } \\
\hline $0-19$ & 20 & 22 & 2 & 44 & $P=0.635$ \\
\hline $20-39$ & 35 & 31 & 14 & 80 & \\
\hline $40-59$ & 12 & 13 & 5 & 30 & \\
\hline $30-39$ & 0 & 3 & 2 & 11 & \\
\hline $60-79$ & 9 & 5 & 1 & 15 & \\
\hline Total & 76 & 71 & 22 & 169 & \\
\hline \multicolumn{6}{|l|}{ Sex } \\
\hline Male & 34 & 34 & 16 & 84 & $P=0.244$ \\
\hline Female & 42 & 37 & 6 & 85 & \\
\hline Total & 76 & 71 & 22 & 169 & \\
\hline \multicolumn{6}{|l|}{ Location } \\
\hline No data & 1 & 4 & 2 & 6 & $P=0.158$ \\
\hline Mandibular & 73 & 62 & 20 & 155 & \\
\hline Maxilla & 2 & 5 & 0 & 7 & \\
\hline Total & 76 & 71 & 22 & 169 & \\
\hline \multicolumn{6}{|l|}{ Size $(\mathrm{cm})$} \\
\hline No data & 4 & 7 & 3 & 14 & $P=0.260$ \\
\hline $0-3$ & 15 & 15 & 5 & 35 & \\
\hline $3.1-6$ & 34 & 38 & 8 & 80 & \\
\hline $6.1-9$ & 11 & 5 & 6 & 22 & \\
\hline$>9$ & 12 & 6 & 0 & 18 & \\
\hline Total & 76 & 71 & 22 & 169 & \\
\hline \multicolumn{6}{|l|}{ Recurrence } \\
\hline No & 71 & 65 & 22 & 158 & $P=0.116$ \\
\hline Yes & 5 & 6 & 0 & 11 & \\
\hline Total & 76 & 71 & 22 & 169 & \\
\hline
\end{tabular}

Association between epithelial WT1 expression and clinicopathological factors examined using chi-square tests.

expression level of WT1 in HAM2 was higher than that in HAM1 and HAM3, it can be presumed that the tumors presented apparent morphological and biological heterogeneity.

In recent years, there has been considerable accumulation of information on the expression of WT1 in tumors of different origins. As these tumors develop from tissues that do not normally express WT1, it has been suggested that its expression may play an oncogenic role in these lesions (4).

In normal tissues, WT1 protein is expressed in the kidneys, uterus, decidua, gonads, ureters, arteries, mesothelium, skeletal muscles, and smooth muscles of the urinary bladder $(18,19)$. Ameloblastomas are odontogenic tumors that develop during the process of tooth formation (odontogenesis). Thus, this study included five tooth germs from different embryological stages that did not exhibit WT1.

Mikami et al. (7) reported that the normal mucosal epithelium did not express WT1; however, WT1 mRNA and protein were expressed in the outer one to three cell layers of infiltrating tumor nests of OSCC. Moreover, WT1 was also expressed in the prickle cell layers of the

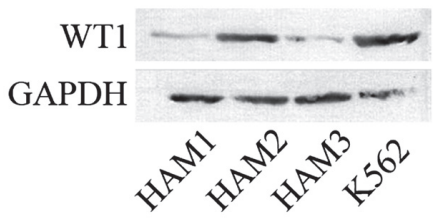

Fig. 4 Western blotting. WT1 protein expression is strongly detected in HAM2 and is minimally detected in HAM1 and HAM3 cell lines. K562 served as a positive control.

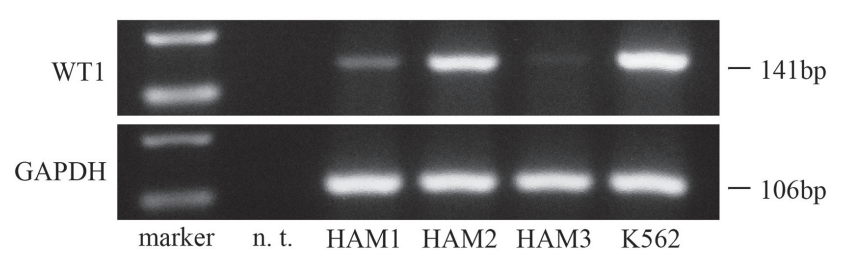

Fig. 5 RT-PCR. WT1 mRNA expression was examined in three cell lines. HAM2 exhibited strong WT1 mRNA expression, HAM1 exhibited moderate expression, and HAM3 weakly expressed WT1. K562 served as a positive control.

epithelium where cell adhesion was weakened to form tumor nests for invasion. The outer layers of an OSCC tumor nest are less differentiated than the inner layer. Langman et al. (20) reported that even though the normal myoepithelium of the salivary gland, which is less differentiated than the glandular epithelium, was negative for WT1 protein, the neoplastic myoepithelium in pleomorphic adenomas of the salivary gland was positive for it. These findings suggest that WT1 is expressed in lower differentiated epithelium instead of higher differentiated epithelium during the process of tumorigenic transformation and neoplastic cells. Kasper et al. (21) reported the transient co-expression of cytokeratins and vimentin in the external enamel epithelium and stellate-reticulum cells during enamel development. Based on these facts, it is suggested that ameloblastomas may be derived from less differentiated epithelium with mesenchymal characteristics, and these reports support the notion that WT1 is related to its pathogenesis.

WT1 protein expression was observed in more than half $(54.7 \%, 92 / 168)$ of all cases included in this study. All of the positive cases exhibited diffuse or granular staining in the cytoplasm, and this finding is consistent 
with those reported by Nakatsuka et al. (6) who examined 494 cases of different human neoplasias including tumors of the urinary and gastrointestinal tract, female and male genital organs, lung, skin, breast, brain, soft tissues and bone using an immunohistochemical approach.

Harada et al. (22) reported that the expression level of WT1 mRNA was higher in more advanced tumors, and Oji et al. (23) reported that WT1 mRNA expression was correlated to the tumor stage of head and neck squamous cell carcinoma.

Ameloblastoma is a benign tumor derived from the odontogenic epithelial component of tooth germ, and presents local invasiveness and a high rate of recurrence if not removed adequately (24). Moreover, ameloblastic carcinoma can arise from a pre-existing benign ameloblastoma (25).

Although the development of non-invasive therapies has been precluded by a lack of understanding of the molecular background of ameloblastoma pathogenesis, Kurppa et al. found a high frequency of BRAF V600E mutations in these tumors (26). Sweeney et al. also reported frequently occurring SMO (encoding Smoothened, SMO) mutations in ameloblastoma, and the effects of these mutations in the activity of the Hedgehog pathway could be inhibited by arsenic trioxide, an anti-leukemia drug (27). Kaye et al. administered BRAFtargeted therapy consisting of dabrafenib at a dosage of $150 \mathrm{mg}$ twice daily and trametinib at a dosage of 2 mg once daily to a stage 4 ameloblastoma patient with a BRAF mutation, and found that the tumor size decreased within 4 days (28).

In the present study, the expression of WT1 was significantly increased in the SA subtype (76.1\%), which presents with the most aggressive characteristics compared to the other variants. Relatively reduced WT1 expression was also noted in the UA variant compared to the SA variant. Clinically, UA does not usually behave as an SA, and does not infiltrate the surrounding bone (24).

The increased expression of WT1 in SA may suggest an association of this protein with tumor biological behavior. In fact, various studies have demonstrated that WT1 gene expression promotes cell growth and motility $(29,30)$ and suppresses apoptosis $(31)$.

Four cases of DA and a case of ameloblastic carcinoma were included in the present study. The DA cases were all negative for WT1, and the case of ameloblastic carcinoma was positive for WT1. However, the number of cases was too small to draw definite conclusions from some of the findings. Since ameloblastomas can transform into ameloblastic carcinomas, further studies are necessary to clarify the correlation between ameloblastic carcinoma and WT1.

After the three major cancer therapies (surgery, chemotherapy, and radiotherapy), cancer immunotherapy is another reliable option available for cancer treatment. In recent years, WT1 peptide-based cancer immunotherapy has been proven to be a new possible cancer treatment, and clinical trials focusing on this have demonstrated effective responses in specific types of cancers (9-15). The complete eradication of cancer stem cells is thought to be essential for curing cancer and, to date, only immunotherapy is capable of killing non-dividing, quiescent cancer stem cells (5). Ameloblastoma is a neoplasm that requires invasive surgery as well as other therapeutic strategies such as immunotherapy.

In conclusion, WT1 overexpression was observed in $76.1 \%$ of SA types, and also in human dental epithelial cell lines derived from the same ameloblastoma patient, suggesting that ameloblastoma presents heterogeneity based on the expression patterns and levels. Our results suggest that the expression of WT1 can influence the pathogenesis of ameloblastoma by varying its expression level in different histological types.

\section{Acknowledgments}

We greatly appreciate the assistance provided by Prof. Alvaro Maglia and Prof. Gabriel Tapia (Histology Department, Universidad de la República, Montevideo, Uruguay) with the tooth germs used as controls. We greatly appreciate the support provided by "Proyectos I+D" and "Equipamientos CSIC", Comisión Sectorial de Investigación Científica (CSIC-UDELAR, Uruguay).

\section{References}

1. Barnes L, Eveson JW, Reichart P (2005) Odontogenic tumours. In: WHO classification of tumours. Pathology and genetics of head and neck tumours, Barnes L, Eveson JW, Reichart P, Sidransky D eds, IARC Press, Lyon, 283-284.

2. Call KM, Glaser T, Ito CY, Buckler AJ, Pelletier J, Haber DA et al. (1990) Isolation and characterization of a zinc finger polypeptide gene at the human chromosome 11 Wilms' tumor locus. Cell 60, 509-520.

3. Oji Y, Ogawa H, Tamaki H, Oka Y, Tsuboi A, Kim EH et al. (1999) Expression of the Wilms' tumor gene WT1 in solid tumors and its involvement in tumor cell growth. Jpn J Cancer Res 90, 194-204

4. Hohenstein P, Hastie ND (2006) The many facets of the Wilms' tumour gene, WT1. Hum Mol Genet 15, Spec 2, R196-201.

5. Sugiyama H (2010) WT1 (Wilms' tumor gene 1): biology and cancer immunotherapy. Jpn J Clin Oncol 40, 377-387.

6. Nakatsuka S, Oji Y, Horiuchi T, Kanda T, Kitagawa M, Takeuchi $\mathrm{T}$ et al. (2006) Immunohistochemical detection of WT1 protein in a variety of cancer cells. Mod Pathol 19, 
804-814.

7. Mikami T, Hada T, Chosa N, Ishisaki A, Mizuki H, Takeda Y (2013) Expression of Wilms' tumor 1 (WT1) in oral squamous cell carcinoma. J Oral Pathol Med 42, 133-139.

8. Qi XW, Zhang F, Wu H, Liu JL, Zong BG, Xu C et al. (2015) Wilms' tumor 1 (WT1) expression and prognosis in solid cancer patients: a systematic review and meta-analysis. Sci Rep 5, 8924.

9. Oka Y, Tsuboi A, Murakami M, Hirai M, Tominaga N, Nakajima $\mathrm{H}$ et al. (2003) Wilms tumor gene peptide-based immunotherapy for patients with overt leukemia from myelodysplastic syndrome (MDS) or MDS with myelofibrosis. Int J Hematol 78, 56-61.

10. Oka Y, Tsuboi A, Taguchi T, Osaki T, Kyo T, Nakajima H et al. (2004) Induction of WT1 (Wilms' tumor gene)-specific cytotoxic T lymphocytes by WT1 peptide vaccine and the resultant cancer regression. Proc Natl Acad Sci U S A 101, 13885-13890.

11. Tsuboi A, Oka Y, Osaki T, Kumagai T, Tachibana I, Hayashi S et al. (2004) WT1 peptide-based immunotherapy for patients with lung cancer: report of two cases. Microbiol Immunol 48, 175-184.

12. Iiyama $\mathrm{T}$, Udaka $\mathrm{K}$, Takeda $\mathrm{S}$, Takeuchi $\mathrm{T}$, Adachi $\mathrm{YC}$, Ohtsuki Y et al. (2007) WT1 (Wilms' tumor 1) peptide immunotherapy for renal cell carcinoma. Microbiol Immunol 51, 519-530.

13. Ohta H, Hashii Y, Yoneda A, Takizawa S, Kusuki S, Tokimasa S et al. (2009) WT1 (Wilms tumor 1) peptide immunotherapy for childhood rhabdomyosarcoma: a case report. Pediatr Hematol Oncol 26, 74-83.

14. Di Stasi A, Jimenez AM, Minagawa K, Al-Obaidi M, Rezvani K (2015) Review of the results of WT1 peptide vaccination strategies for myelodysplastic syndromes and acute myeloid leukemia from nine different studies. Front Immunol 6, 36.

15. Mayanagi S, Kitago M, Sakurai T, Matsuda T, Fujita T, Higuchi $\mathrm{H}$ et al. (2015) Phase I pilot study of Wilms tumor gene 1 peptide-pulsed dendritic cell vaccination combined with gemcitabine in pancreatic cancer. Cancer Sci 106, 397-406.

16. Hatakeyama S, Mizusawa N, Tsutsumi R, Yoshimoto K, Mizuki H, Yasumoto S et al. (2011) Establishment of human dental epithelial cell lines expressing ameloblastin and enamelin by transfection of hTERT and cdk 4 cDNAs. J Oral Pathol Med 40, 227-234.

17. Bologna-Molina R, Mosqueda-Taylor A, Molina-Frechero N, Mori-Estevez AD, Sánchez-Acuña G (2013) Comparison of the value of PCNA and ki-67 as markers of cell proliferation in ameloblastic tumors. Med Oral Patol Oral Cir Bucal 18, e174-179.

18. Park S, Schalling M, Bernard A, Maheswaran S, Shipley GC, Roberts D et al. (1993) The Wilms tumour gene WT1 is expressed in murine mesoderm-derived tissues and mutated in a human mesothelioma. Nat Genet 4, 415-420.

19. Ramani P, Cowell JK (1996) The expression pattern of Wilms' tumour gene (WT1) product in normal tissues and paediatric renal tumours. J Pathol 179, 162-168.

20. Langman G, Andrews CL, Weissferdt A (2011) WT1 expression in salivary gland pleomorphic adenomas: a reliable marker of the neoplastic myoepithelium. Mod Pathol 24, 168-174.

21. Kasper M, Karsten U, Stosiek P, Moll R (1989) Distribution of intermediate-filament proteins in the human enamel organ: unusually complex pattern of coexpression of cytokeratin polypeptides and vimentin. Differentiation 40, 207-214.

22. Harada Y, Nonomura N, Nishimura K, Tamaki H, Takahara S, Miki T et al. (1999) WT1 gene expression in human testicular germ-cell tumors. Mol Urol 3, 357-364.

23. Oji Y, Inohara H, Nakazawa M, Nakano Y, Akahani S, Nakatsuka S et al. (2003) Overexpression of the Wilms' tumor gene WT1 in head and neck squamous cell carcinoma. Cancer Sci 94, 523-529.

24. Gardner DG, Heikinheimo K, Shear M, Philipsen HP, Coleman H (2005) Ameloblastomas. In: WHO classification of tumours. Pathology and genetics of head and neck tumours, Barnes L, Eveson JW, Reichart P, Sidransky D eds, IARC Press, Lyon, 296-300.

25. Sciubba JJ, Eversole LR, Slootweg PJ (2005) Odontogenic / ameloblastic carcinomas. In: WHO classification of tumours. Pathology and genetics of head and neck tumours, Barnes L, Eveson JW, Reichart P, Sidransky D eds, IARC Press, Lyon, 287-289.

26. Kurppa KJ, Catón J, Morgan PR, Ristimäki A, Ruhin B, Kellokoski J et al. (2014) High frequency of BRAF V600E mutations in ameloblastoma. J Pathol 232, 492-498.

27. Sweeney RT, McClary AC, Myers BR, Biscocho J, Neahring L, Kwei KA et al. (2014) Identification of recurrent SMO and BRAF mutations in ameloblastomas. Nat Genet 46, 722-725.

28. Kaye FJ, Ivey AM, Drane WE, Mendenhall WM, Allan RW (2014) Clinical and radiographic response with combined BRAF-targeted therapy in stage 4 ameloblastoma. J Natl Cancer Inst 107, 378.

29. Tsuboi A, Oka Y, Ogawa H, Elisseeva OA, Tamaki H, Oji Y et al. (1999) Constitutive expression of the Wilms' tumor gene WT1 inhibits the differentiation of myeloid progenitor cells but promotes their proliferation in response to granulocytecolony stimulating factor (G-CSF). Leuk Res 23, 499-505.

30. Jomgeow T, Oji Y, Tsuji N, Ikeda Y, Ito K, Tsuda A et al. (2006) Wilms' tumor gene WT1 17AA(-)/KTS(-) isoform induces morphological changes and promotes cell migration and invasion in vitro. Cancer Sci 97, 259-270.

31. Ito K, Oji Y, Tatsumi N, Shimizu S, Kanai Y, Nakazawa T et al. (2006) Antiapoptotic function of 17AA(+)WT1 (Wilms' tumor gene) isoforms on the intrinsic apoptosis pathway. Oncogene 25, 4217-4229. 